Research Article

\title{
Probabilistic Assessment of Damage from Same Shock Response Spectra due to Variations in Damping
}

\author{
Arup Maji (i) \\ Org 1557, Sandia National Laboratories, Albuquerque, NM, USA \\ Correspondence should be addressed to Arup Maji; amaji@sandia.gov
}

Received 14 April 2020; Revised 6 August 2020; Accepted 15 November 2020; Published 28 November 2020

Academic Editor: Matteo Strozzi

Copyright (C) 2020 Arup Maji. This is an open access article distributed under the Creative Commons Attribution License, which permits unrestricted use, distribution, and reproduction in any medium, provided the original work is properly cited.

\begin{abstract}
Interpretation of field data from shock tests and subsequent assessment of product safety margins via laboratory testing are based on the shock response spectra (SRS). The SRS capture how a single degree of freedom (SDOF) structure responds to the shock at differing frequencies and, therefore, no longer contain the duration or other temporal parameters pertaining to the shock. A single duration can often be included in the technical specification or in the recreation of acceleration vs. time history from the specified SRS; however, there is little basis for that beyond technical judgment. The loss of such temporal information can result in the recreated SRS being the same while its effect on a system or component can be different. This paper attempts to quantify this deficiency as well as propose a simple method of capturing damping from shock waves that can allow the original waveform to be more accurately reconstructed from the SRS. In this study the decay rate associated with various frequencies that comprise the overall shock was varied. This variation in the decay rate leads to a variation in the acceleration vs. time history, which can be correlated to a "Damage Index" that captures the fatigue damage imparted to the object under shock. Several waveforms that have the same SRS but varying rates of decay for either high- or low-frequency components of the shock were investigated. The resulting variation in stress cycles and Damage Index is discussed in the context of the lognormal distribution of fatigue failure data. It is proposed that, along with the SRS, the decay rate is also captured to minimize the discrepancy between field data and representative laboratory tests.
\end{abstract}

\section{Introduction}

Mechanical vibration and shock have a detrimental effect on components or systems. Therefore, shock tests are conducted to ensure items can survive the repeated shocks that they are expected to endure during their lifetime. The level of shock to be applied in the shock tests is prescribed in terms of the shock response spectra (SRS). The SRS levels used for testing are typically prescribed in the terms of P99 or P95 levels (for example, P99 implies that the shock applied in the test will exceed $95 \%$ of the shock the item may see in its lifetime).

An issue that arises with this approach is that SRS are not a unique representation of shock; various shock events can have the same SRS. The different shocks (decaying acceleration vs. time) corresponding to the same SRS will inflict a different amount of damage to the item of interest. In this study, the effect of 4 shocks (Trials 1-4) that have identical
SRS was investigated to quantify the consequence in such variations. The 4 shocks were generated by varying the decay rate (damping) of the underlying shock acceleration vs. time.

The SRS quantify the input shock, and "damping" relates to the decay rate of the input shock. Damage, on the contrary, is a consequence of the shock applied. The consequence of the shock can be measured in terms of a "Damage Index". In this paper, a "Damage Index" is quantified in terms of the magnitude and number of stress cycles as per equation (1). Its absolute value is significant only when a specific material/structure is being considered and the stress cycles needed to cause failure are known. In the context of this paper, the absolute value of the "Damage Index" is not significant, and only the relative differences between Trial 1 through Trial 4 are significant. Damping (of the input shock) is constant for each frequency but varies among the 5 frequencies and from Trial 1 to Trial 4. 
Statistical data are available in the literature on how the Damage Index at failure (\# of stress cycles leading to failure) varies among tests. This information can be used to assess how a variation in the number and magnitude of stress cycles relates to the probability of failure. This, in turn, allows the variation in stress cycles from the 4 trials to be related to the consequent variation in the probability of failure. The various sources of data and the analytical methods are presented in the next section.

\section{Implications of Fatigue Test Data}

In this section, test data from several sources in the literature were examined for variation in fatigue damage from test data, as defined by the standard deviation of the $\log _{10}$ (damage).

The basic relationship between cyclic stress $(\Delta S)$ and number of cycles to failure $(N)$ is provided by Basquin's power law [1], also referred to as the Miner-Palmgren relation [2]:

$$
\Delta S^{\beta} N=C
$$

where the constant $C$ can be thought of as a "Damage Index" or measure of accumulated damage leading to failure. The dimensional units of this parameter $C$ are dependent on those of the underlying parameter ( $\Delta S$ has units of stress, typically in $\mathrm{MPa}$, and $N$ is unitless) and hence can be converted to other units accordingly. The unitless parameter $\beta$ is determined from test data using linear regression on a semilog scale ( $\beta=6.66$ was used in this paper).

Paris and Erdogan [3] provided an analogous expression or slow fatigue crack growth per cycle $\delta a / \delta N$ as a function of the stress intensity factor $(K)$ instead of stress and the material constant $A$ replacing $C$ in the Miner-Palmgren relation:

$$
\frac{\delta a}{\delta N}=A \Delta K^{m}
$$

These relations form the basis for statistical analyses of fatigue data for various material-structural systems $[4,5]$ and standard practices used in the industry. Equation (1) is also expressed in the following form:

$$
\begin{aligned}
\log N & =\frac{1}{\beta} \log \left(\frac{C}{\Delta S}\right) \text { or } \log (\text { damage }) \\
& =\log (C)=\log (N)+\beta \log (\Delta S) .
\end{aligned}
$$

The standard deviations of the quantities are thus related by the following equation:

$$
\sigma(\text { damage })=\sigma(\log N)+\beta \sigma(\log \Delta S) .
$$

Szala and Szala [6] investigated the fatigue life of D16CzATW aluminum alloy using fatigue test data on structural elements subject to different types of cyclic loads. Using a fatigue model based on equation (1), they arrived at a mean fatigue life parameter $N c$ from 6 tests. The normalized (to the mean) fatigue life parameter was calculated, and the standard deviation of $\log$ (normalized-Nc) was determined to be $10.4 \%$ (Table 1 ).
TABle 1: Mean number of fatigue life cycles from test data from Szala and Szala [6].

\begin{tabular}{lcc}
\hline Test \# & Normalized $(N c)$ & $\log (N c)$ \\
\hline 1 & 1.24 & 0.094 \\
2 & 0.75 & -0.128 \\
3 & 0.91 & -0.043 \\
4 & 0.75 & -0.125 \\
5 & 1.08 & 0.034 \\
6 & 1.27 & 0.105 \\
Mean & & -0.010 \\
St. deviation & & $\mathbf{1 0 . 4 \%}$ \\
\hline
\end{tabular}

Leonetti et al. [7] fit fatigue test data to the Miner-Palmgren cumulative damage law and looked at the deviations for butt weld joints and cover-plated beams of steel for a large number $(>500)$ of tests. Based on the standard deviation $\sigma_{w}$ of $\log _{10} N$, the standard deviation of fatigue damage was determined to be $29 \%$ and $19 \%$, respectively, for the butt joint and cover plates tested.

Peng et al. [8] investigated fatigue failure under combinations of small and large amplitude cyclic loading with cumulative damage defined as the summation of damage for each type of loading. Damage for each type of load was defined in terms of the Miner-Palmgren relation. The damage for various load combinations was normalized to their corresponding mean values and evaluated using a lognormal distribution, as shown in Table 2, which summarizes the variation in total damage for 3 types of metals. The standard deviation of the lognormal distribution shown in Table 2 was determined to be $14.7 \%, 12.3 \%$, and $11.4 \%$, respectively.

Shen [9] provided an extensive database of fatigue failure of the aluminum 2023-T4 alloy. Figure 1 shows the data on a log-log scale and the best-fit straight line. Using the inverse of the slope of this line as the measure of $\beta(1 / 0.11=9.1)$, damage was calculated for each data point. The standard deviation of $\log$ (damage) was determined to be 0.27 . It can be noted in Figure 1 that the scatter in data is much higher for the low-stress cycles. Therefore, this analysis was repeated using only the data $\geq 371.7 \mathrm{MPa}(\log \Delta S \geq 2.57)$, as shown in Figure 2. The slope of the line has changed to 0.18 and $\beta=5.57$. The standard deviation of $\log$ (damage) is now only $3.7 \%$ due to the lower scatter (note the small stress range for these data). Figure 3 shows how the damage remains constant in spite of the variation in the stress range due to the corresponding changes in the number of cycles to failure.

Overall, the standard deviation of log(damage) varies from $10 \%$ to $30 \%$, and smaller standard deviations are possible when the range of stress cycles is small. Therefore, the analyses in the next section will be based on the assumptions of the standard deviation $\sigma\left(\log _{10}\right.$ damage $)=10 \%$ to $30 \%$. A comprehensive literature survey of fatigue test data is beyond the scope of this paper; the reader can use the analyses presented, henceforth, to assess the consequences for any fatigue test data relevant to their specific application. 
TABle 2: Fatigue damage data for various materials by Peng et al. [8].

\begin{tabular}{|c|c|c|c|c|c|}
\hline \multicolumn{2}{|c|}{ 300CVM steel } & \multicolumn{2}{|c|}{ Aluminum-2024-T42 } & \multicolumn{2}{|c|}{ 30NiCrMoV12 steel } \\
\hline Normalized damage & Log(damage) & Normalized damage & Log(damage) & Normalized damage & Log(damage) \\
\hline 0.76 & -0.121 & 0.85 & -0.072 & 0.58 & -0.238 \\
\hline 0.82 & -0.087 & 0.78 & -0.106 & 0.78 & -0.107 \\
\hline 1.03 & 0.012 & 0.69 & -0.164 & 0.83 & -0.083 \\
\hline 1.22 & 0.086 & 0.64 & -0.192 & 0.80 & -0.095 \\
\hline 1.03 & 0.011 & 0.66 & -0.178 & 0.74 & -0.133 \\
\hline 1.61 & 0.207 & 0.70 & -0.153 & 1.04 & 0.019 \\
\hline 1.11 & 0.045 & 0.84 & -0.074 & 0.91 & -0.041 \\
\hline 1.30 & 0.114 & 0.74 & -0.133 & 0.69 & -0.162 \\
\hline 1.10 & 0.043 & 0.88 & -0.057 & 0.86 & -0.068 \\
\hline 0.46 & -0.341 & 1.18 & 0.072 & 1.17 & 0.069 \\
\hline 0.51 & -0.296 & 1.24 & 0.095 & 1.27 & 0.105 \\
\hline 0.77 & -0.112 & 1.25 & 0.098 & 1.54 & 0.187 \\
\hline 1.05 & 0.023 & 1.39 & 0.144 & 1.07 & 0.028 \\
\hline 1.45 & 0.160 & 1.40 & 0.147 & 1.28 & 0.106 \\
\hline 0.90 & -0.046 & 1.29 & 0.109 & 1.41 & 0.150 \\
\hline 0.76 & -0.120 & 1.26 & 0.100 & 1.08 & 0.035 \\
\hline \multirow[t]{2}{*}{1.13} & 0.055 & 1.20 & 0.080 & 0.95 & -0.022 \\
\hline & & 1.16 & 0.064 & 0.85 & -0.072 \\
\hline Standard deviation & $14.7 \%$ & & $12.3 \%$ & & $11.4 \%$ \\
\hline
\end{tabular}

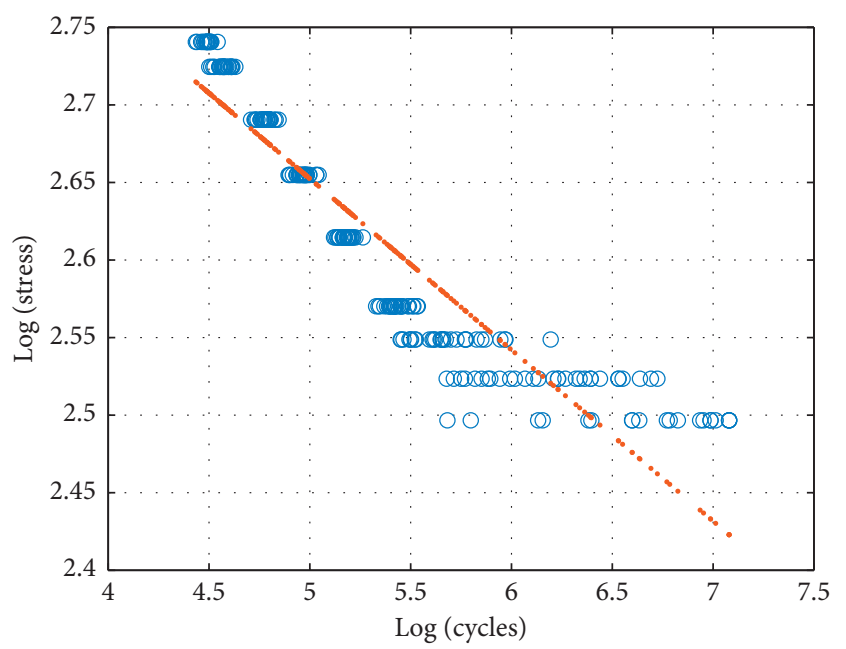

FIGURE 1: Linear fit to fatigue failure test data (entire stress range).

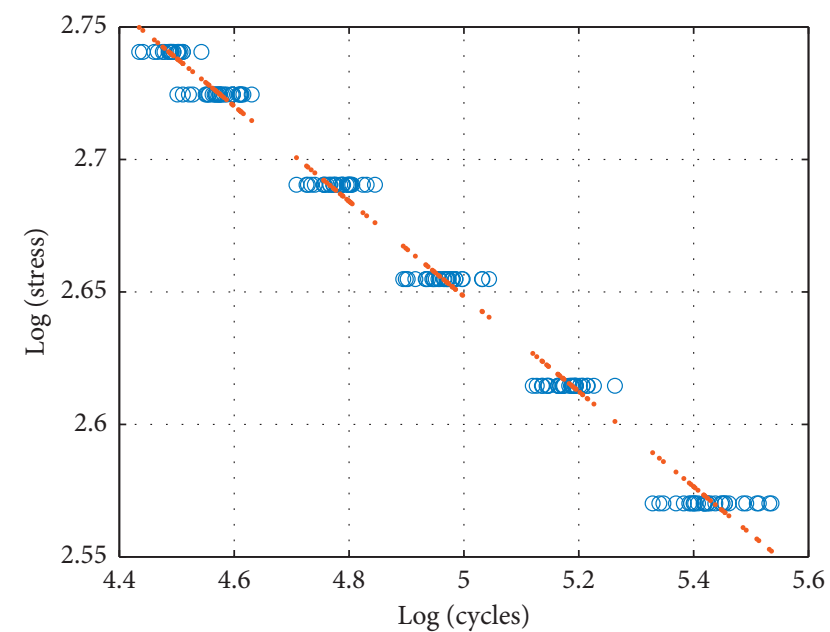

Figure 2: Linear fit to fatigue failure test data (higher stress range).

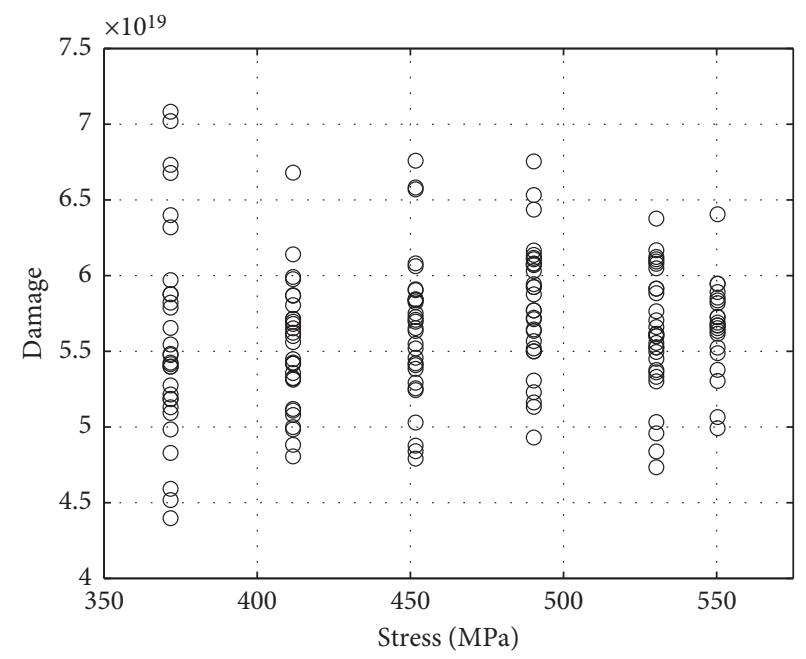

Figure 3: Variation in damage for various stress ranges.

\section{Damage Corresponding to Different Decay Rates for the Same SRS}

Shock testing specifications are based on the concept of the shock response spectra (SRS) which no longer contains the time history. The SRS were originally conceived to address the need to convey the effect of earthquake damage to civil infrastructure $[10,11]$ in a concise manner. The SRS are typically based on the maxi-max absolute acceleration (MMAA) of the response of an SDOF (single degree of freedom) structure to the shock acceleration vs. time. As a result, the SRS do not contain any information on the decay rate of the original wave. Different component frequencies in a shock wave may attenuate at different rates. For instance, the high-frequency component typically attenuates faster; however, the decay at different frequencies is also dependent on through what media or structure the shock wave has been 
transmitted. Therefore, the issues addressed in this paper pertain to the following.

Damage Index (DI) is defined in the context of Miner's rule for low-cycle fatigue damage as discussed earlier (parameter $C$ in equation (1)). For the same SRS, how is the imparted "Damage Index" (C) affected by the varying decay rate of the different component frequencies? An implicit part of this question is the determination of what causes differences in SRS and DI as the decay rate changes. The value of the material-dependent exponent $\beta$ was chosen to be 6.66 .

While this paper studies the influence of damping as an input parameter to shock and consequent damage, damping has also been extensively studied as a consequence of damage. Kouris et al. [12] investigated the variation in modal damping as a measure of structural damage by applying progressively greater vibration load to a building leading to "'near-collapse damage state." Variation in damping was proven to be a better measure of structural damage than the shift in natural frequency/modes [13].

3.1. Effect of Damping on the SRS. Four different acceleration time-histories with the same SRS were investigated. These acceleration vs. time data $\ddot{x}(t)$ were generated using decayed sines (equation (5)), where $\omega$ is the frequency in radians and $\xi$ is damping. The exponential decay parameter $\alpha$ represents the final amplitude/initial amplitude ratio after a certain time period. Five sine waves (at 100, 200, 400, 650, and $950 \mathrm{~Hz}$ ) were added to create the total acceleration time history. Trial 1 had the amplitude of each of the 5 sine waves (acc 1-5) set to 10. As shown in Table 3, the damping of each frequency $\xi$ was inversely proportional to the frequency as per equation (5). The exponential decay parameter $\alpha$ determines how the sine waves decay as per equation (5). Therefore, since $\xi \omega=\alpha$ was constant for each of the 5 constituent sine waves, each of them decay to the same level at the end of the $0.08 \mathrm{sec}$ duration under consideration. Figure 4 shows 3 of the 5 sine waves each decaying to the same amplitude after $0.08 \mathrm{sec}$.

$$
\begin{aligned}
\ddot{x}(t) & =\sin \omega t e^{-\xi \omega t}, \quad \text { where } \xi \omega \\
& =\alpha=\text { Constant Exp Decay. }
\end{aligned}
$$

For Trial 2, the damping of the 2 highest frequencies (650 and $950 \mathrm{~Hz}$ ) was increased by a factor of 3 . As a result, these waves decayed faster (Figure 5). However, the amplitude of these two components was increased (Table 3 ) such that the resulting SRS were identical to that of Trial 1. Figure 5, therefore, shows a higher amplitude for the $950 \mathrm{~Hz}$ signal (blue); note that the actual peak is somewhat less than that of the table because of the rapid decay of the high-frequency content.

For Trial 3, the damping of the 2 highest frequencies ( 650 and $950 \mathrm{~Hz}$ ) was increased by a factor of 4 relative to Trial 1 . Once again, the amplitude of these two components was increased (Table 3) such that the resulting SRS were identical to that of Trial 1. The SRS of Trials 1-3 are shown in Figure 6. The 2 waveforms (Trial 1 and Trial 2) are shown side by side in Figure 7. Due to the increase in the amplitude of the highfrequency component, Trial 2 had a higher maximum
TABLE 3: Synthesis of four waveforms with the same SRS.

\begin{tabular}{lccccc}
\hline Frequency (Hz) & 100 & 200 & 400 & 650 & 950 \\
\hline Trial 1: proportional damping to get the same decay & \\
\hline Damping & 0.07500 & 0.03750 & 0.01875 & 0.01154 & 0.00750 \\
\hline Amplitude & 10 & 10 & 10 & 10 & 10 \\
\hline Trial 2: $X 3$ & damping & of Trial & 1 at 650 and $950 \mathrm{~Hz}$ & \\
\hline Damping & 0.075 & 0.0375 & 0.01875 & 0.03462 & 0.0225 \\
\hline Amplitude & 10 & 10 & 10 & 13.6 & 13.2 \\
\hline Trial 3: $X 4$ & damping of Trial & 1 at 650 and $950 \mathrm{~Hz}$ \\
\hline Damping & 0.075 & 0.0375 & 0.01875 & 0.04616 & 0.03 \\
\hline Amplitude & 10 & 10 & 10 & 15.4 & 14.7 \\
\hline Trial & $4:$ & $X 2$ & damping of Trial & 1 at 100 and $200 \mathrm{~Hz}$ \\
\hline Damping & 0.15 & 0.075 & 0.01875 & 0.01154 & 0.0075 \\
\hline Amplitude & 14 & 14 & 10 & 10 & 10 \\
\hline
\end{tabular}

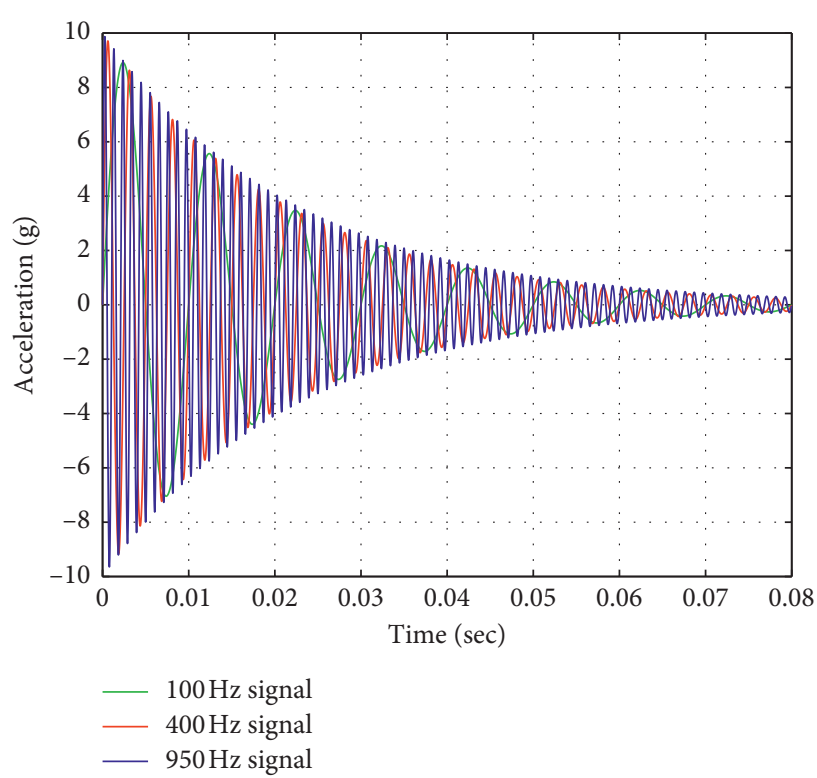

Figure 4: Accelerations at 100, 400, and $950 \mathrm{~Hz}$ decaying to the same final amplitude.

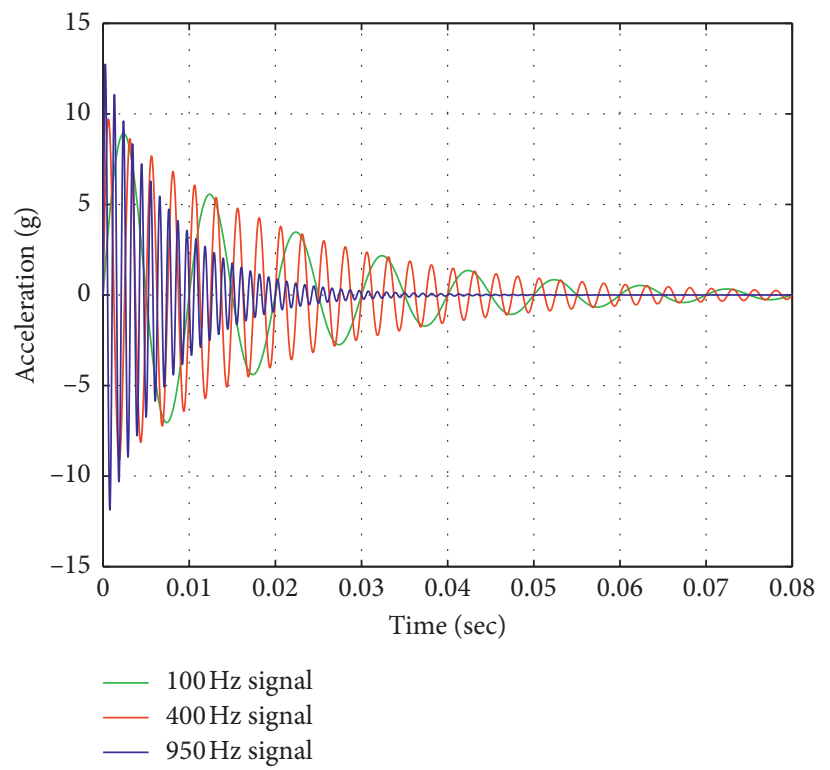

Figure 5: Accelerations at $950 \mathrm{~Hz}$ decaying faster than 100 and $400 \mathrm{~Hz}$. 


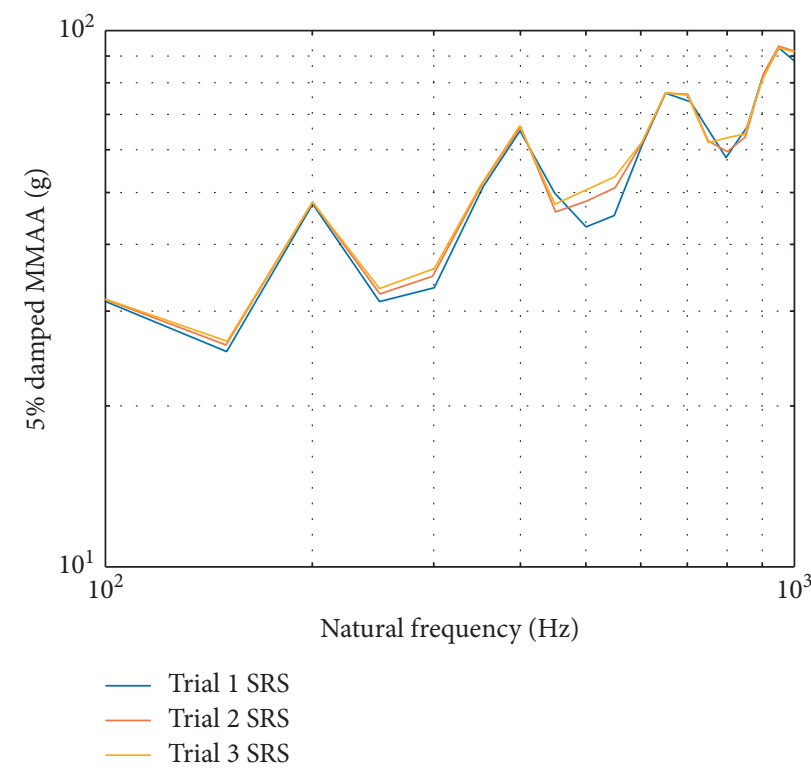

FigurE 6: SRS of waveforms from Trial 1, Trial 2, and Trial 3.

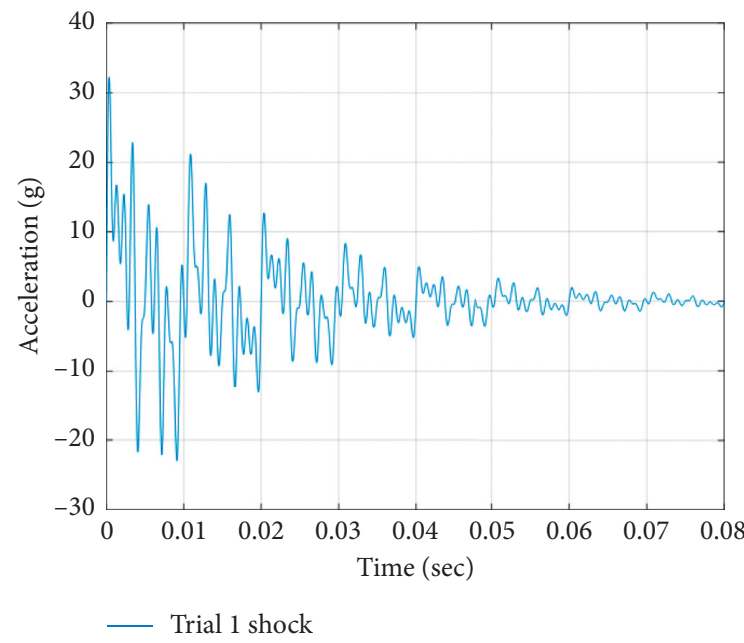

(a)

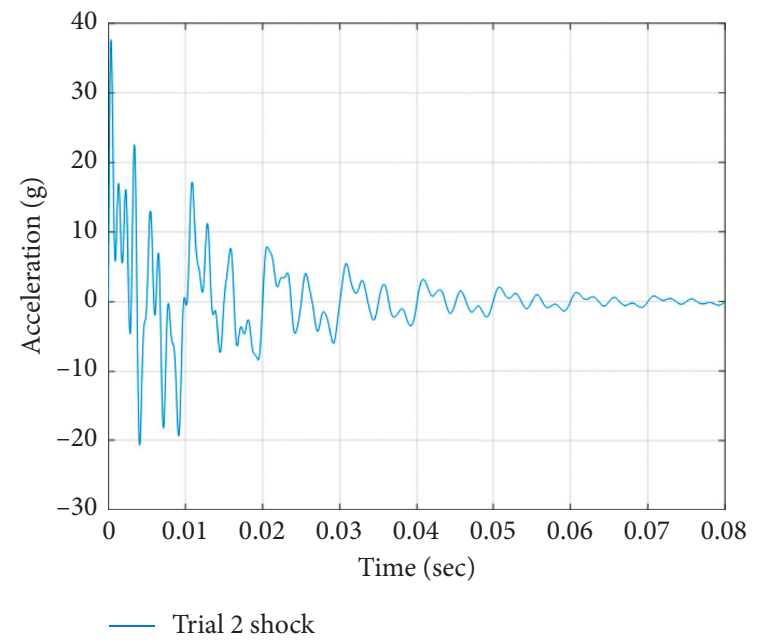

(b)

Figure 7: Waveforms from Trial 1 vs. Trial 2.

acceleration, which contributed to a greater Damage Index as will be discussed next.

The SRS were computed using the MMAA after the following convolution integral that captures the response of a single degree of freedom (SDOF) spring-mass structure $\ddot{y}(t)$ due to input acceleration $\ddot{x}(t)$ shown in the following equation, where $\omega$ is the natural frequency of the SDOF structure and $\xi$ is the damping constant:

$$
\ddot{y}(t)=\frac{-\omega}{\sqrt{1-\xi^{2}}} \int_{0}^{t} \ddot{x}(\tau) e^{-\xi \omega(t-\tau)} \sin \quad \omega \sqrt{1-\xi^{2}}(t-\tau) \mathrm{d} \tau .
$$

Next, using the rain flow count algorithm in Matlab ${ }^{\mathrm{TM}}$, the \# and amplitude of cycles ( $N$ and $\sigma$ in equation (1)) were determined from the acceleration vs. time such as those shown in Figure 7. The results for all 4 trials are shown in Figure 8. Using these data and equation (1), the Damage Index (DI) was determined to be $2.90 \times 10^{11}, 3.39 \times 10^{11}$, $3.99 \times 10^{11}$, and $3.24 \times 10^{11}$ for Trial 1 through Trial 4 , respectively. The approximately $17 \%$ increase in DI for Trial 2 is due to the higher initial peak in the shock wave leading to more high-stress cycles (50-60); note that Trial 2 has a smaller \# of cycles at the stress range of $0-10$. The contribution of low-stress cycles to the DI is minimal due to the exponent $\beta=6.66$ in the equation for DI. Likewise, the $38 \%$ increase in DI for Trial 3 is due to the high-stress cycle (60-70) which was not present for Trial 1.

For Trial 4, the damping of the two lowest frequencies was increased by a factor of 2 (Table 3). Figure 9 shows the 


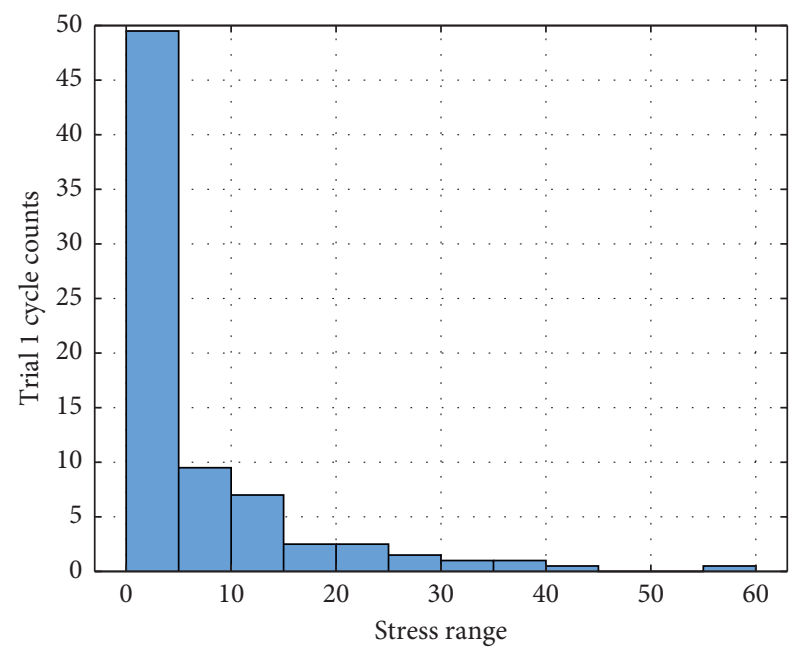

Cumulative damage $=2.90 \mathrm{e} 11$

(a)

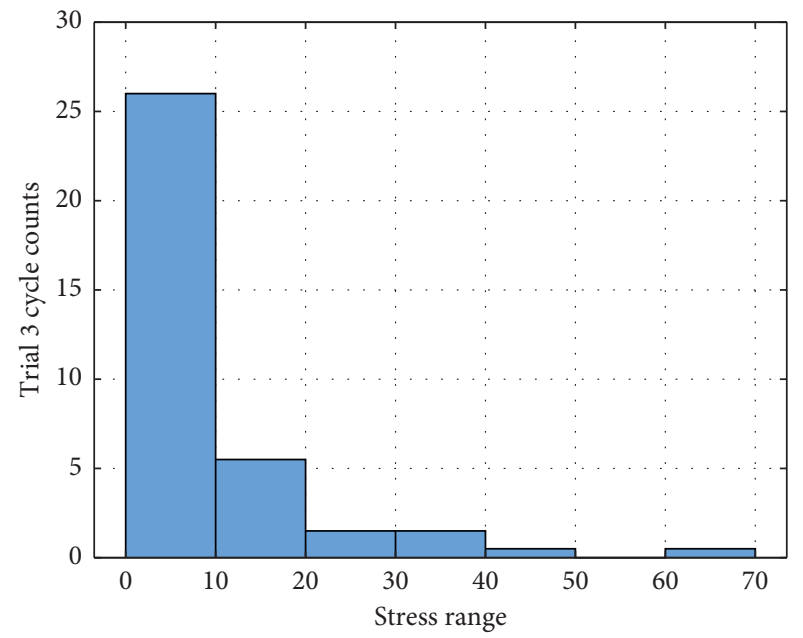

Cumulative damage $=3.99 \mathrm{e} 11$

(c)

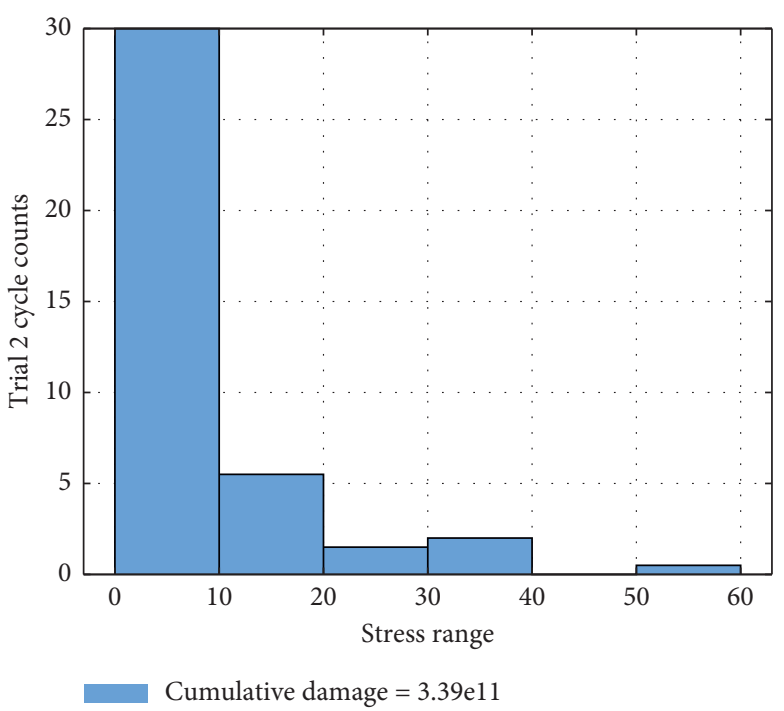

(b)

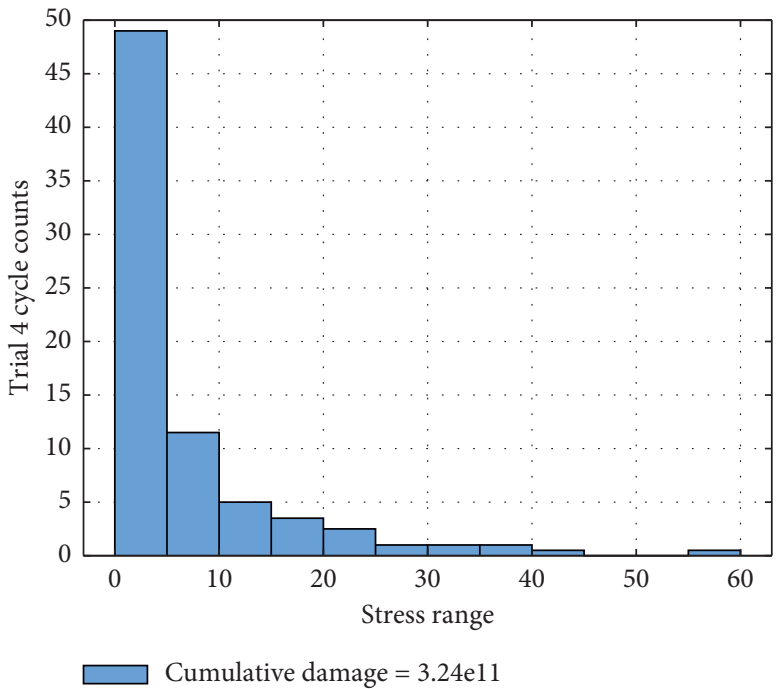

(d)

Figure 8: Stress range $(\sigma)$ and corresponding cycle count $(n)$ from 4 trials.

resulting shock wave and the rapidly decayed $100 \mathrm{~Hz}$ component, which required the amplitude at that frequency to increase (to keep the same SRS). This led to the DI increasing to $3.24 \times 10^{11}(12 \%$ higher than that of Trial 1$)$.

\section{Consequence of Damage Variations in Test Reliability Levels}

4.1. Probability of Exceeding Damage Threshold. The previous sections showed the following:

(i) Different acceleration vs. time data from the same SRS can lead to differences in the "Damage Index."

(ii) Variations in materials and structures result in a variation in the "Damage Index" at failure (for static loads, this is analogous to failure of any material at different stress levels).
This section provides a discussion on how a variation in the fatigue-based "Damage Index" in the previous section affects the overall assessment of the probability failure. We will use the concept of P99 level as the probability of failure less than $1 \%$. Conducting a shock test to P99 level implies that the test exceeds the real-life environment $99 \%$ of the time. So, when there is a $20 \%$ variation in the Damage Index from the different recreations of acceleration vs. time from the same SRS (based on the range of $12 \%$ to $38 \%$ variations in DI discussed earlier), how does that affect the probability of failure? This issue will be investigated in the next section considering the effect of $20 \%$ variation in the fatigue damage imparted during a test.

First, lognormal statistics is discussed briefly in terms of natural $\log ($ base $e)$, defined as $y=\ln (x)$, where $x$ is lognormal if $y$ follows the normal distribution. Closed-form equations provided below provide some valuable insights that can then 


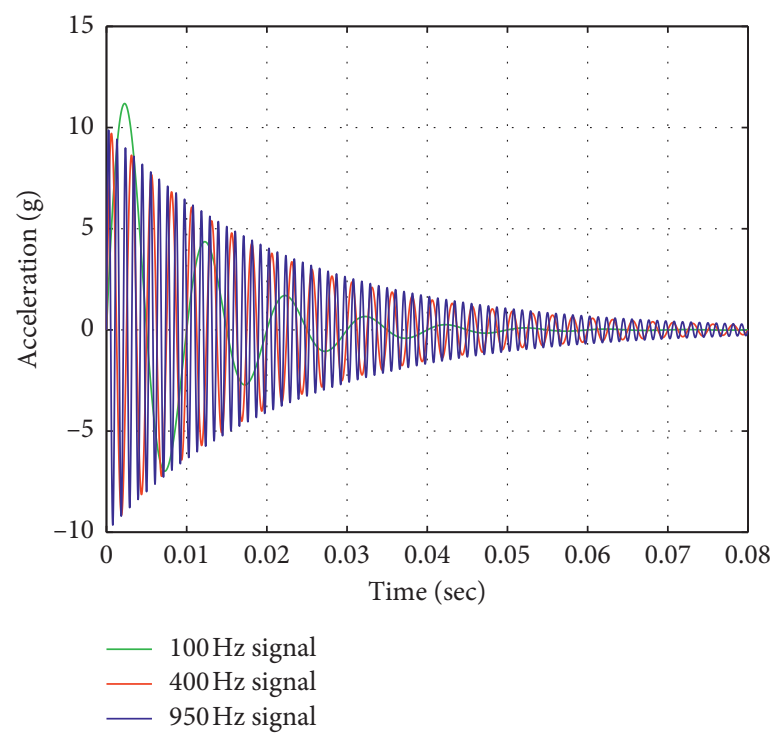

(a)

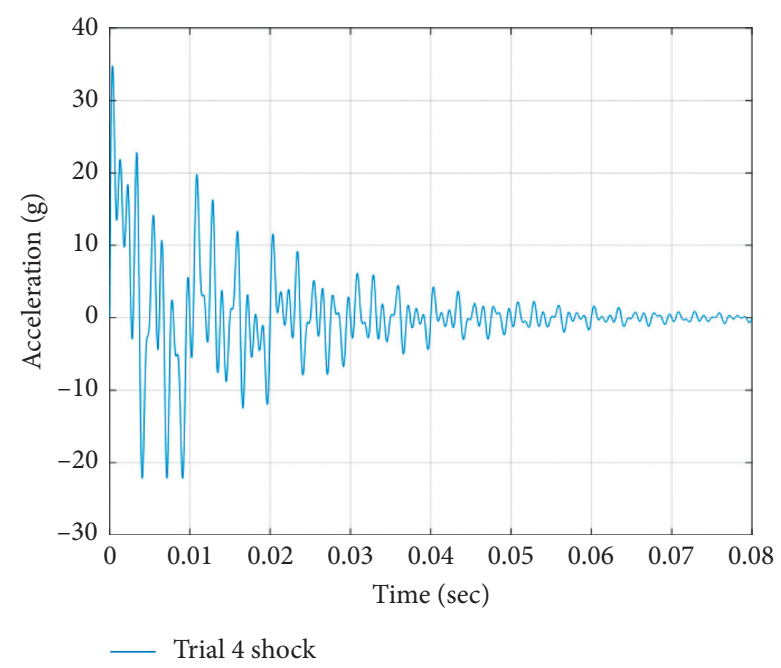

(b)

Figure 9: Trial 4: amplified peak at $100 \mathrm{~Hz}$ component and overall signal.

be extended to the definition of lognormal statistics $y=\log 10(x)$. If the mean and standard deviation of $y$ is $\mu$ and $\sigma$, the mean and standard deviation of $x\left(=e^{y}\right)$ is $\mu^{\prime}$ and $\sigma^{\prime}$. Equations for probability distribution function (PDF) and cumulative PDF (CDF) are also provided as follows:

$$
\begin{gathered}
\text { mean of } x: \mu^{\prime}=e^{\left(\mu+\sigma^{2} / 2\right)}, \\
\text { standard deviation of } x: \sigma^{\prime}=\sqrt{\left[e^{\sigma^{2}}-1\right] e^{\left(2 \mu+\sigma^{2}\right)}}, \\
\text { coefficient of variation in } x: \frac{\sigma^{\prime}}{\mu^{\prime}}=\sqrt{\left[e^{\sigma^{2}}-1\right]},
\end{gathered}
$$

probability distribution function $(\mathrm{PDF}): \operatorname{PDF}(x)$

$$
=\frac{1}{x \sigma \sqrt{2 \pi}} e^{\left(-(\ln (x)-\mu)^{2} / 2 \sigma^{2}\right)},
$$

cumulative PDF (CDF): $\operatorname{CDF}(x)=\frac{1}{2}+\frac{1}{2} \operatorname{erf}\left[\frac{\ln (x)-\mu}{\sqrt{2} \sigma}\right]$.

As per equation (8), the coefficient of variation in $x$ is independent of the mean $\mu$ of the underlying data $y$. Consequently, a change in $\mu$ only leads to a stretching of the $\mathrm{CDF}$, which is also evident from equation (10) since a change in $\mu$ is offset by a corresponding change in $\ln (x)=y$. Figure 10 shows this for a simulation using $10^{6}$ randomly generated values of $y$ (using MatLab ${ }^{\mathrm{TM}}$ randn) from which $x$ is computed $\left(=e^{y}\right)$. The PDF and CDF (vs. $x$ ) are shown for $\sigma=0.25$ and $\mu=0$, and 1. The larger $\mu(=1)$ shows a larger spread in the lognormal data as well as a shift in the mean value to the right. The CDF shows that the $50 \%$ values for both equal $x=e^{\mu}(=1.0$ and 2.72 , respectively; red lines). If the value of $x$ is increased by $20 \%$ to 1.2 and 3.26 (red dotted lines), the corresponding CDFs remain the same (0.77). Hence, a proportionate change in $x$ leads to the same change in CDF. This will also be observed when the definition is changed to $\log 10(x)=y$.

In order to investigate how the Matlab ${ }^{\mathrm{TM}}$ simulation of statistical distribution converges with an increase in the number of data (varied from $10^{3}$ to $10^{7}$ ), the theoretical values of the mean and standard deviation of $x\left(\mu^{\prime}\right.$ and $\left.\sigma^{\prime}\right)$ were obtained using equation (7) and normally distributed $y$ (with $\mu=0$ and $\sigma=1$ ). These were then compared with the mean and standard deviation of $x$ based on the Matlab ${ }^{\mathrm{TM}}$ random number generator to create $y$. Figure 11 shows how this ratio converges to 1.0 for $10^{7}$ data points. Henceforth, simulations were conducted with $10^{7}$ data points.

4.2. Simulation of the Effect of a 20\% Change in C on Probability of Failure. The simulation involved the following steps.

The PDF and CDF of $10^{7}$ data points were generated using $\mu=0$ and varying $\sigma$ (0.1 to 0.3 ).

Using the CRF, the value of $x$ corresponding to a $1 \%$ probability of failure ( $1 \%$ probability of the material's fatigue life being below that of the fatigue damage corresponding to the SRS) was determined. Note that this corresponds to the P99 level (99\% probability of the material's fatigue life being higher than that of the fatigue damage corresponding to the SRS).

For a $20 \%$ increase in $x$ (i.e., $1.2 x$ ), the probability of failure was determined. For instance, if the new probability of failure corresponding to $1.2 x$ is $5 \%$, it means that the $20 \%$ increase in $x$ resulted in the P99 changing to P95. Figure 12 shows the probability of failure for different combinations of $\mu$ and $\sigma$ (10 simulations for each). Note that an increase in $\sigma$ from 0.25 to 1.0 reduces the probability of failure. This is because with increased scatter in the fatigue strength of the material, a $20 \%$ change in the damage imparted is less 


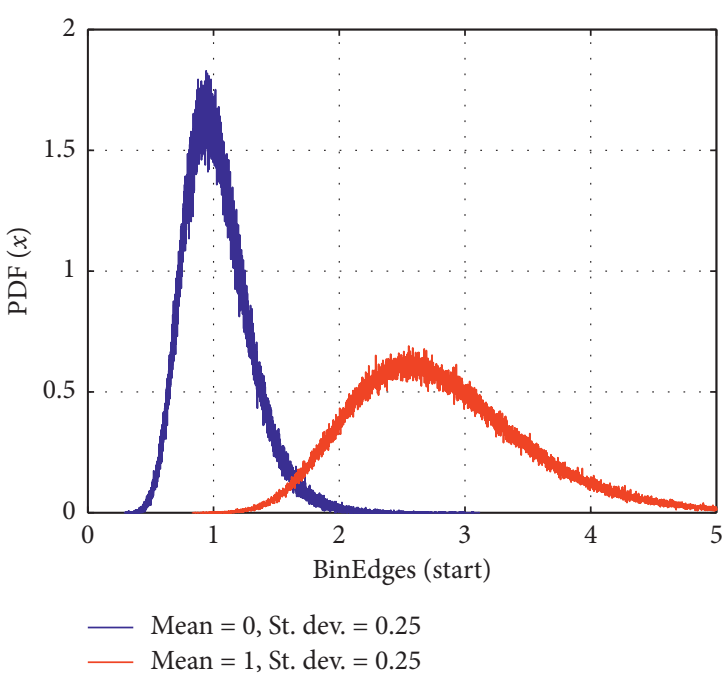

(a)

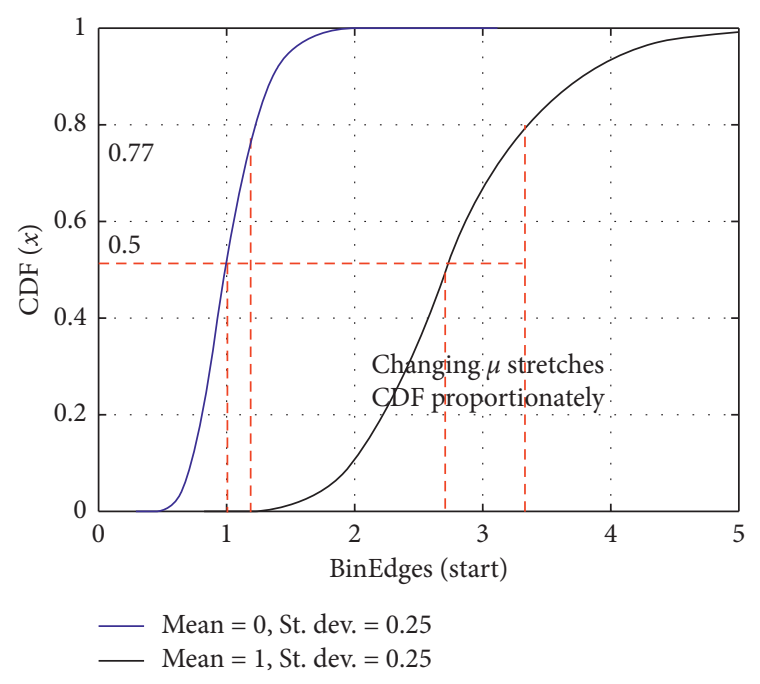

(b)

FIGURE 10: PDF and CDF with changing mean (0 and 1) and same standard deviation.

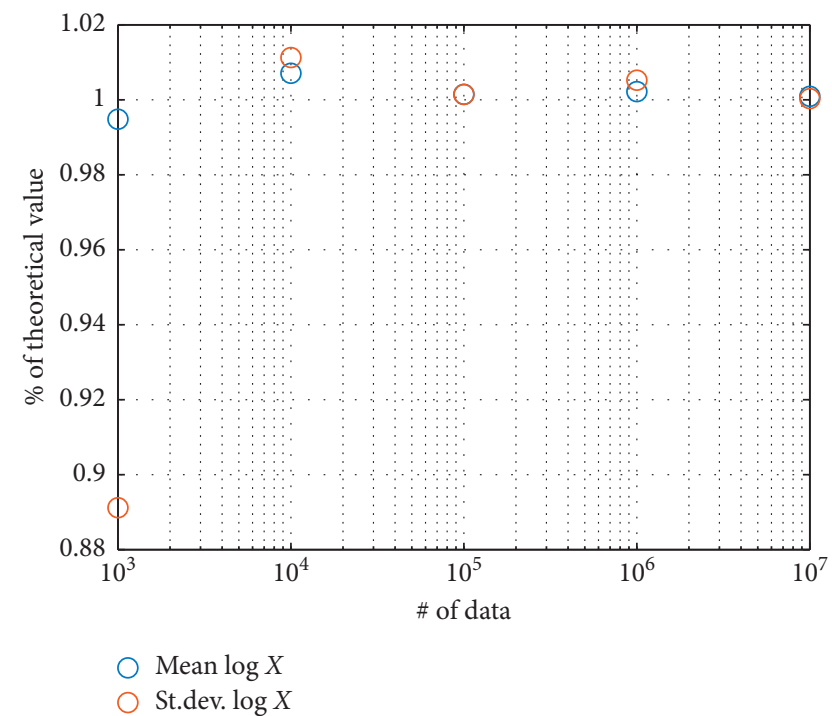

FIGURE 11: Convergence of mean and standard deviation to theoretical values.

consequential. Note that the change in the mean value is inconsequential due to reasons discussed in the previous section (coefficient of variation is independent of the mean).

Therefore, the remaining simulations (10 runs each) were done with $\mu=0$ and varying $\sigma$ from 0.1 to 0.3 ; results are shown in Figure 13. As the scatter in the material's fatigue strength decreases (decreasing $\sigma$ ), the consequence of the $20 \%$ change in $x$ is progressively more consequential. For $\sigma=0.1$, P99 changes to worse than P94, while for $\sigma=0.3$, P99 changes only to P98.

\section{Capturing Rate of Decay of Shock}

A method is suggested for capturing the decay rate associated with the different component frequencies. This would allow

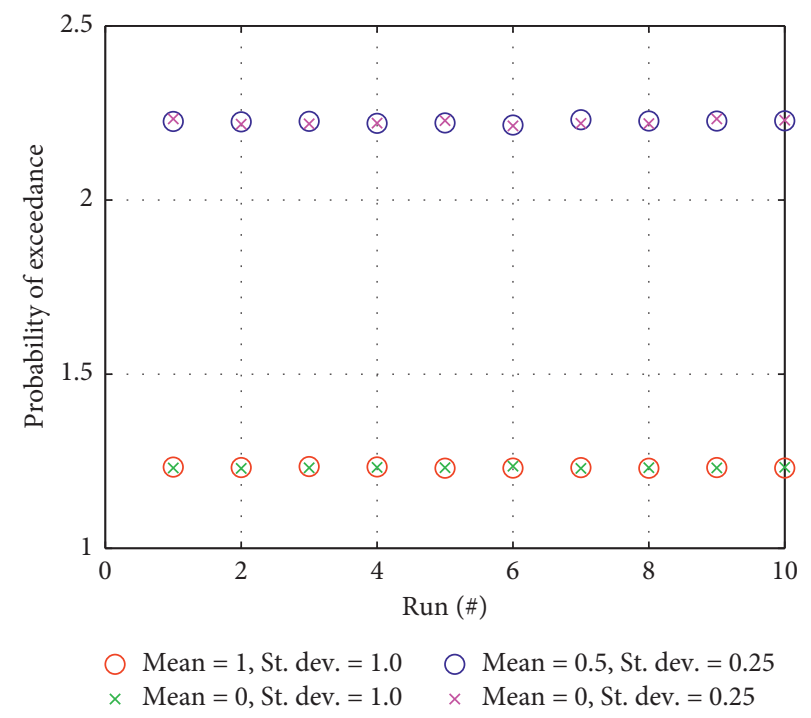

Figure 12: Probability of failure (damage exceeding material capacity).

the original waveform to be replicated more accurately. The SRS at each frequency are a function of both the amplitude and damping associated with that frequency. The same is also true for the FFT of a decaying shock wave. As the shock wave decays with time, the amplitude of the FFT decreases; this can be utilized to capture the damping at each frequency.

The input signal at each frequency $\omega$ is a decaying sine wave of amplitude $A$ enveloped by an exponential decay. The decaying envelope $I$ of each sine wave can be characterized by the damping parameter $\xi$ according to the following equation:

$$
I=A e^{-\xi \omega t}
$$

Integrating equation (11) from the start to time $T$, one obtains the mean value of the envelope over the time period 


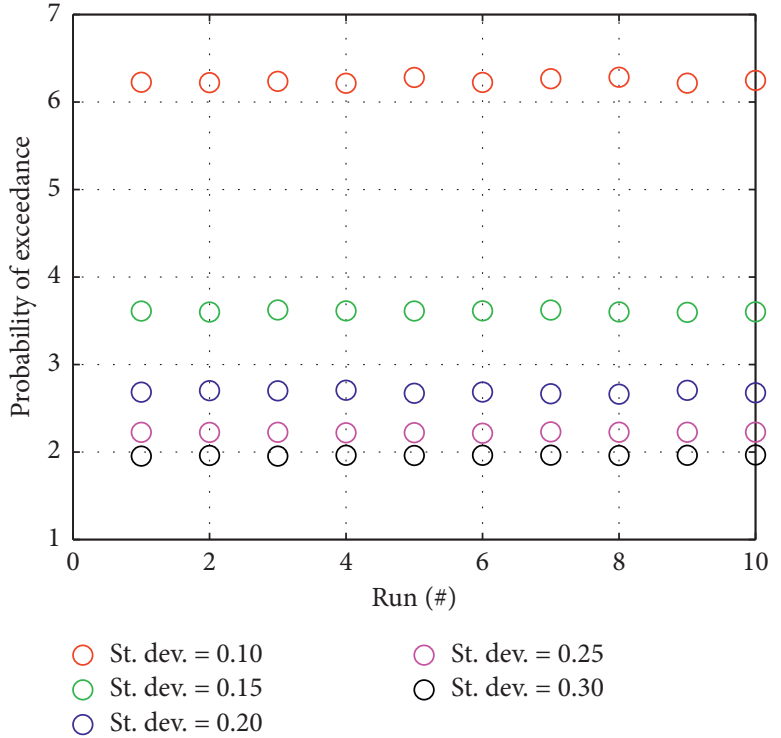

(a)

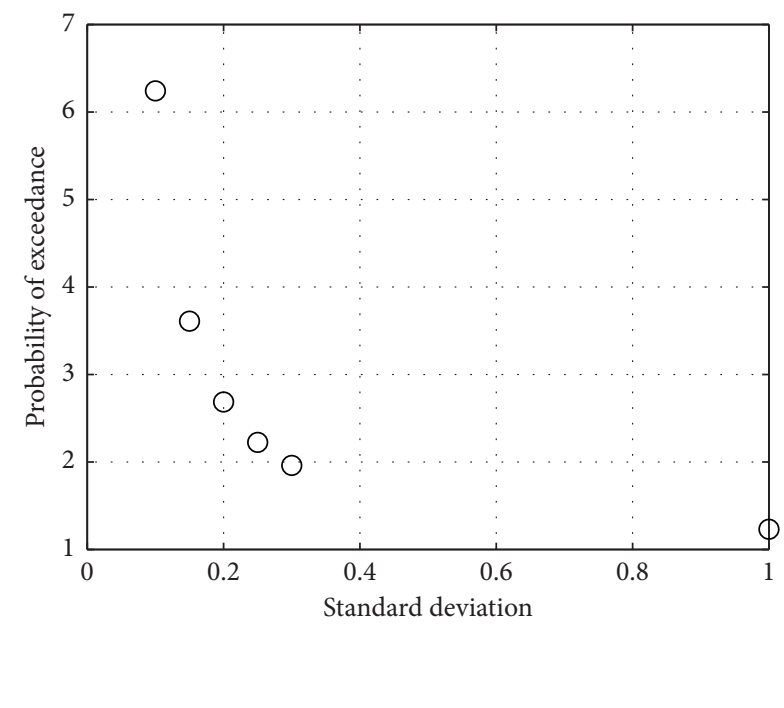

(b)

FIgURE 13: Probability of failure (damage exceeding material capacity).

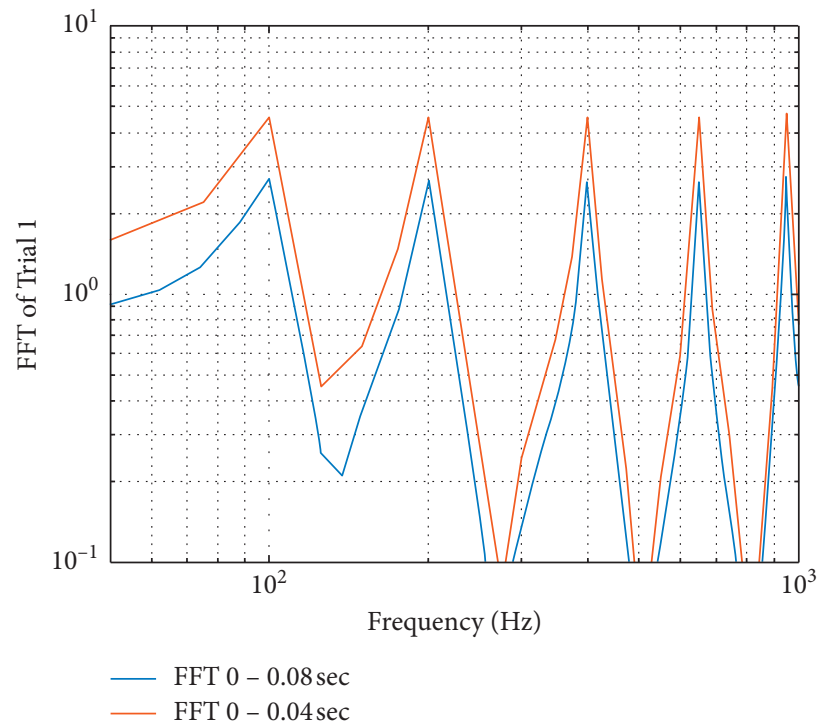

FIgURE 14: FFT of Trial 1 signal (full and 1/2 duration).

$[0, T]$, as shown in equation (2). This mean value represents the amplitude of the Fourier transform of the decaying sine wave over this interval $(\mathrm{P} / \mathrm{A}<1$ because of the decay):

$$
P=\frac{A}{T} \int_{0}^{T} e^{-\xi \omega t} \mathrm{~d} t=\frac{A}{\xi \omega T}\left(1-e^{-\xi \omega T}\right) .
$$

Equation (12) has two unknowns: amplitude $A$ and damping $\xi$ of the sine wave and, therefore, needs to be evaluated at two different points. Therefore, by conducting a fast Fourier transform (FFT) of the shock wave at two different time intervals $\left[0, T_{1}\right]$ and $\left[0, T_{2}\right)$, one can obtain two corresponding values of the mean amplitude of the sine waves $P_{1}$ and $P_{2}$. Based on equation (2), equation (3) represents these two specific situations. The unknown damping $\xi$ is then evaluated by numerically solving the following equation:

$$
\frac{1-e^{-\xi \omega T_{1}}}{1-e^{-\xi \omega T_{2}}}=\frac{P_{1} T_{1}}{P_{2} T_{2}}
$$

Figures 14 and 15 show the FFT of the input signal from Trial 1 and Trial 2, respectively, from 0 to $0.08 \mathrm{sec}$ and from 0 to $0.04 \mathrm{sec}$, respectively. The lower numbers for $0-0.08 \mathrm{sec}$ are due to the greater decay over the full duration. The peak values in Figure 14 are the same for both durations due to proportional damping and constant amplitude of each sine wave. The peak values in Figure 15 are lower for the 2 high 


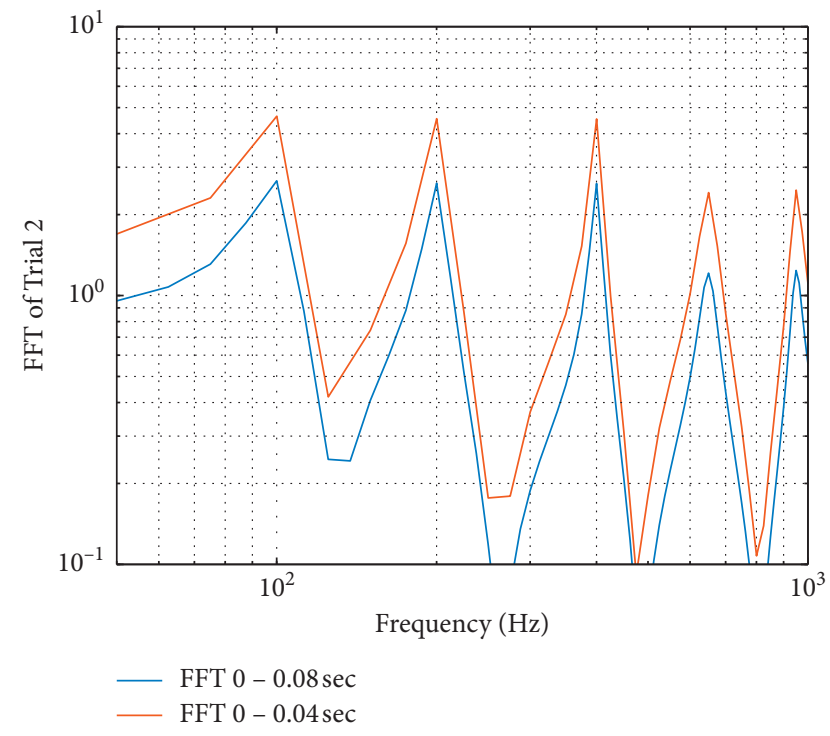

FIgURE 15: FFT of Trial 2 signal (full and 1/2 duration).

TABLE 4: Comparison of actual decay rates with those computed from FFT.

\begin{tabular}{lccccc}
\hline & \multicolumn{5}{c}{ Frequencies } \\
& $100 \mathrm{~Hz}$ & $200 \mathrm{~Hz}$ & $400 \mathrm{~Hz}$ & $650 \mathrm{~Hz}$ & $950 \mathrm{~Hz}$ \\
\hline Trial 1 actual & 0.07500 & 0.03750 & 0.01875 & 0.01154 & 0.00750 \\
Trial 1 from FFT & 0.07499 & 0.03750 & 0.01875 & 0.01154 & 0.00750 \\
Trial 2 actual & 0.075 & 0.0375 & 0.01875 & 0.03462 & 0.0225 \\
Trial 2 from FFT & 0.0756 & 0.0376 & 0.0188 & 0.0346 & 0.0222 \\
Trial 3 actual & 0.075 & 0.0375 & 0.01875 & 0.04616 & 0.03 \\
Trial 3 from FFT & 0.0757 & 0.0377 & 0.0189 & 0.0496 & 0.0280 \\
Trial 4 actual & 0.15 & 0.075 & 0.01875 & 0.01154 & 0.0075 \\
Trial 4 from FFT & 0.1433 & 0.0741 & 0.0188 & 0.0115 & 0.0075 \\
\hline
\end{tabular}

frequencies due to the larger damping; the values for the full duration are even lower. The damping values determined using the methodology described above are shown in Table 4; both the actual and the estimated damping values are provided for comparison. The method captures the damping values very accurately for all 4 trials. The only time the estimated damping is slightly different (shaded orange) is when the duration of $0.04 \mathrm{sec}$ does not include sufficient waves to be statistically representative.

\section{Conclusions}

(i) Assuming longer duration equates to a smaller damping for the same SRS. This leads to a lower Damage Index (DI) and unconservative test (highstress cycles are eliminated).

(ii) An increase in damping leads to an increase in DI (approximately 17\% and 38\% for Trial 2 and Trial 3 compared to Trial 1) due to an increase in peak acceleration values. A few high cycles can dominate the Damage Index due to the exponent $\beta$. (iii) An increase in DI can significantly impact the estimated reliability of the test for realistic lognormal statistics of fatigue test data observed in tests of different materials. A perceived $\mathrm{P} 99$ test can become a P95 test or worse.

(iv) The influence of an increase in DI on reliability is less if the underlying data correlating DI to failure have a larger scatter (higher $\sigma$ ). Large variation in material property makes the accuracy of the test specification less consequential. The analysis process presented in this paper provides a methodology to evaluate the appropriate level of rigor and accuracy for shock test specification.

(v) The coefficient of variation in a lognormal distribution is independent of the mean of the underlying normal distribution. This implies that the mean fatigue damage $\mu$ is of no consequence to the issue (changes in the PXX level) discussed in this paper.

(vi) Using the Fourier transform of shock signals over two different durations, it is possible to compute the damping associated with the frequencies of interest with high accuracy. This would allow original waveforms to be captured more accurately vs. just the SRS.

\section{Data Availability}

All data in the paper unless explicitly cited and referenced were generated in this study by the author.

\section{Disclosure}

Sandia National Laboratories is a multimission laboratory managed and operated by National Technology and Engineering Solutions of Sandia, LLC, a wholly owned subsidiary of Honeywell International Inc., for the U.S. Department of Energy's National Nuclear Security Administration under contract DE-NA0003525. This paper describes objective technical results and analysis. Any subjective views or opinions that might be expressed in the paper do not necessarily represent the views of the U.S. Department of Energy or the United States Government.

\section{Conflicts of Interest}

The authors declare that they have no conflicts of interest.

\section{References}

[1] O. H. Basquin, The Exponential Law of Endurance Tests, American Society for Testing Materials, Phildelphia, PA, USA, 1910.

[2] M. A. Miner, "Cumulative damage in fatigue," Journal of Applied Mechanics, vol. 3, pp. 159-164, 1945.

[3] P. Paris and F. Erdogan, "A critical analysis of crack propagation laws," Journal of Basic Engineering, vol. 85, no. 4, pp. 528-533, 1963.

[4] ASTM, ASTM Practice for Statistical Analysis of Linear or Linearized Stress-Life $(S-N)$ and Strain-Life $(\varepsilon-N)$ Fatigue 
Data', ASTM E-739-91, ASTM, West Conshohocken, PA, USA, 1998.

[5] J. Brozzetti, M. A. Hirt, I. Ryan, G. Sedlacek, and I. Smith, Background Information on Fatigue Design Rules-Statistical Evaluation-Chapter 9-Document 9.01, ECCS, Colorado Springs, CO, USA, 1989.

[6] J. Szala and G. Szala, "A fatigue life calculation method for structural elements made of D16CzATW aluminum alloy," Polish Maritime Research, vol. 17, no. 3, 2010.

[7] D. Leonetti, S. B. Hashemi, D. Allaix, and J. Maljaars, "Fatigue damage evaluation using S-N curves obtained by different data fitting methods," in Proceedings of the 39th IABSE Symposium, Vancouver, Canada, September 2017.

[8] Z. Peng, H.-Z. Huang, J. Zhou, and Y.-F. Li, "A new cumulative fatigue damage rule based on dynamic residual S-N curve and material memory concept," Metals, vol. 8, no. 6, p. 456, 2018.

[9] C. Shen, The Statistical Analysis of Fatigue Data, University Microfilms International, Ann Arbor, MI, USA, 1994.

[10] C. Lalanne, "Shock and vibration," in Vibration and Shock Handbook, C. De Silva, Ed., McGraw-Hill, New York, NY, USA, 2005.

[11] J. Edward Alexander, "Shock response spectrum-a primer," Sound and Vibration, vol. 43, no. 6, pp. 5-14, 2009.

[12] L. A. S. Kouris, A. Penna, and G. Magenes, "Seismic damage diagnosis of a masonry building using short-term damping measurements," Journal of Sound and Vibration, vol. 394, pp. 366-391, 2017.

[13] L. A. S. Kouris, A. Penna, and G. Magenes, "Dynamic modification and damage propagation of a two-storey fullscale masonry building," Advances in Civil Engineering, vol. 2019, Article ID 2396452, 21 pages, 2019. 